\title{
The Effect of BPA on Reed Root Exudates
}

\author{
Lin Wang ${ }^{1,}$, ${ }^{\text {Li W Wang }}{ }^{2, b}$ and Chunxiao Sun ${ }^{1}$ \\ ${ }^{1}$ School of environment science and Technology, Ocean University of China, Shandong 250001, \\ China. \\ 2 School of yyy, Harbin Institute of Technology, Heilongjiang 150001, China. \\ alwangouc@126.com, bliwanghit@126.com
}

\begin{abstract}
The metabonomics method was employed to detect and analyze the exudates of reed root under BPA stress. PCA(Principal component analysis)and OPLS-DA(Orthogonal partial least squares discriminant analysis) used to identify the main potential metabolites variation with and without BPA addition, 64 compounds were found,including a variety of small molecule glucose, organic acid, amino acid and other small molecule compounds. 14 compounds of main metabolites found by OPLS-DA Loading diagram, VIP prediction diagram and analysis of variance had close relationship with tolerance and degradation of BPA, mainly including glucose, organic acids, amino acids and alcohols.
\end{abstract}

Keywords: BPA (bisphenol-A), reed, exudates.

\section{Introduction}

Root exudates are general name for a series of carbon metabolites and the secondary metabolites that are resistant to infection and stimulate or enhance their resistance[1][2]. Bio-transformation and bio-degradation are the main mechanisms for the restoration of aromatic compounds, microbial activity can be stimulated by releasing root exudates under stress, and transformation and degradation of microorganisms will be promoted[3]. The root exudates play very important role on restoration of plants, the function and path of exudates was explored recently[4],especially under different environment, some specific components, such as phenolic compounds, glucose, dissolved organic carbon and so on, vary with outside pressure[5][6][7],However, there are few studies on the specific components and changes of root exudates in wetland plants under endocrine disrupting chemicals stress.

In this paper, the composition and changes of root exudates in reed under bisphenol-A stress were analyzed by the method of metabolomics based on gas chromatography-mass spectrometry (GC-MS), and the composition of root exudates which were related to the removal of bisphenol-A was selected to enhance the removal efficiency of bisphenol-A by tidal flow constructed wetland.

\section{Materials and Methods}

Metabonomics is a method for measuring dynamic multiple parameters of quantitative biological systems under external stimulation and interference[8][9]. Based on molecular, metabolomics find the relationship between changes in biological metabolic patterns and various life phenomena, and have been used in many areas[8],[10][11][12], Gas chromatography-mass spectrometry (GC-MS) can be used to analyze small molecules, such as amino acids, sugars, organic acids and fatty acids, so it is the most mature method in the analysis and application of plant metabolites[13][14].

\subsection{Plants and Culture Condition}

60 reed seedlings with health and uniform root system were selected, cultured at lab for 3 months with Hoagland nutrient solution(components:45mg/L Ca(NO3)2•4H2O; 506 mg/L KNO3; 80 mg/L NH4NO3; 136 mg/L KH2PO4; 493 mg/L MgSO4; 27.8 mg/L FeSO4•7H2O; 37.3 mg/L NaEDTA). The nutrient solution was changed every 3days, $\mathrm{pH}$ was justified by $0.1 \mathrm{~mol} / \mathrm{L}$ of $\mathrm{NaOH}$, or $\mathrm{HCl}$ to 6.5. After the culture finished, 20 strains of reed with good and uniform growth were selected and transplanted to $20500 \mathrm{~mL}$ aseptic bottles for 10 days ( 1 plants / bottles), the ambient environment in this period was kept at $18-26^{\circ} \mathrm{C}$ and natural light. When the plants growth were stable, all the 
experimental equipment was sterilized at high temperature and washed with sterile water for 3 times, $500 \mathrm{~mL}$ ultra pure water was added to each aseptic bottle, 5 reeds of 20 reeds prepared above were used as blank groups (without BPA) for another 3days culture; 5 reeds were used as the control group for 3 days BPA treatment, and 10mg of BPA was added to the culture solution, BPA concentration of culture solution was $20 \mathrm{mg} /$; 5 reeds were used as the blank group after 6days culture without BPA adding, and 5reeds as control group after 6days treatment with BPA treatment.

\subsection{The Collection of Root Exudates}

After 3days culture, the roots of 10 reeds samples was washed 3times with distilled water and washed with sterile water for 3 5 times, and each of 10 reeds samples were transplanted to $100 \mathrm{mLsterile}$ bottle filled with $50 \mathrm{ml}$ ultra pure water, root exudates released to pure water for $6 \mathrm{~h}$, $10 \mathrm{ml}$ ultra pure water with root exudates was taken from $50 \mathrm{ml}$ ultra pure water above, and freezedried for 2 days by the vacuum freeze drying at $-80 \mathrm{C}$. Freeze-dried residues were added to $10 \mathrm{~mL}$ of pre-cool methanol, root exudates extracted and blown dry with nitrogen blows. Residue was dissolved in $1 \mathrm{~mL}$ hexane and standby. The collection of the root exudates of the 6days treatment was in the same way.

\subsection{GC-MS Analysis}

The derivatization process is as follows: first, $40 \mathrm{~L} 20 \mathrm{mg} / \mathrm{mL}$ of methoxamine hydrochloride solution (solvent: pyridine) was added into the sample, mixed for $2 \mathrm{~h}$ at $37 \mathrm{oC}$, and then $70 \mathrm{~L} \mathrm{~N}$-methylN-trifluoroacetamide (MSTFA) added, mixed for 30min at 37oC; After the reaction was completed, the $1 \mathrm{~mL}$ sample was filtered through the $0.45 \mathrm{~m}$ filter membrane to the vial for GC-MS analysis.

A Thermo TR5-MS capillary column (30mx0.32 mmx0.25 mu m) was used for GC-MS analysis. Operation condition and temperature program are shown in the Table 1. AMDIS(Automated Mass Spectral Deconvolution \&Identification System) and Met-IDEA was employed to analyze the GCMS figure, the components were determined qualitatively by comparing with NIST.05 database, and determined quantitative by measuring the peak area of each component.

Table 1.Operating conditions for GC-MS analysis

\begin{tabular}{cc}
\hline items & condition \\
\hline Injection port temperature & $230^{\circ} \mathrm{C}$ \\
Injection mode & $(50: 1,0.75 \mathrm{~min})$ \\
Sample quantity & $1 \mu \mathrm{L}$ \\
Flow rate of carrier gas & $1 \mathrm{~mL} / \mathrm{min}($ constant flow $)$ \\
temperature program & $70^{\circ} \mathrm{C}(1 \mathrm{~min}) \rightarrow 76^{\circ} \mathrm{C}\left(1{ }^{\circ} \mathrm{C} / \mathrm{min}\right) \rightarrow 330^{\circ} \mathrm{C}\left(5^{\circ} \mathrm{C} / \mathrm{min}, 10 \mathrm{~min}\right)$ \\
Binding port temperature & $250^{\circ} \mathrm{C}$ \\
Ion scanning range & $50-600 \mathrm{~m} / \mathrm{z}$ \\
Electron energy of EI mode & $70 \mathrm{eV}$ \\
Solvent retention time & $3 \mathrm{~min}$ \\
\hline
\end{tabular}

\subsection{Data Processing}

The peak area of each component detected by GC-MS was input into the Excel, and standardized processing, the results were analyzed by PCA (Principal component analysis)and OPLSDA(Orthogonal partial least squares discriminant analysis), the deviation phenomena of samples treated by different condition was explained, The bio-markers were predicted and identified by VIP(VIP>1), and ANOVA(analysis of variance) performed at same time. 


\section{Results and Discussion}

\subsection{GC-MS Analysis}

A total of 64 compounds were detected by GC-MS analysis of reed, including a variety of small molecules of saccharide (such as RNA, fructose and mannose, etc.), organic acid (such as malic acid, succinic acid, etc.), amino acid (such as: L-valine, L-tyrosine, L-alanine, etc.), alcohols (such as: sorbitol, xylitol, inositol, etc.), and other small molecule compounds, such as: oleic acid and palmitic acid and dehydroabietic acid, etc. The same sample was injected 6times, the relative standard deviation of the each sample calculated, which exhibited the low error.

\subsection{The Root Exudats Analysis}

After root exudates identification finished, the processed data was input into the SIMCA-P software, and converted into PCA and OPLS-DA figure. The results of PCA and OPLS-DA of reeds are shown in Figure 1 and Figure2.

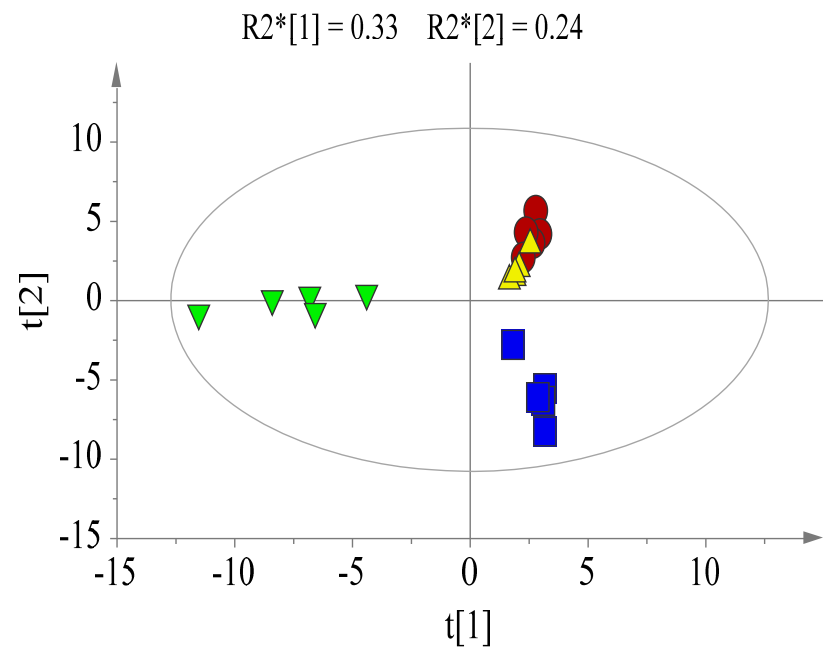

(a) PCA

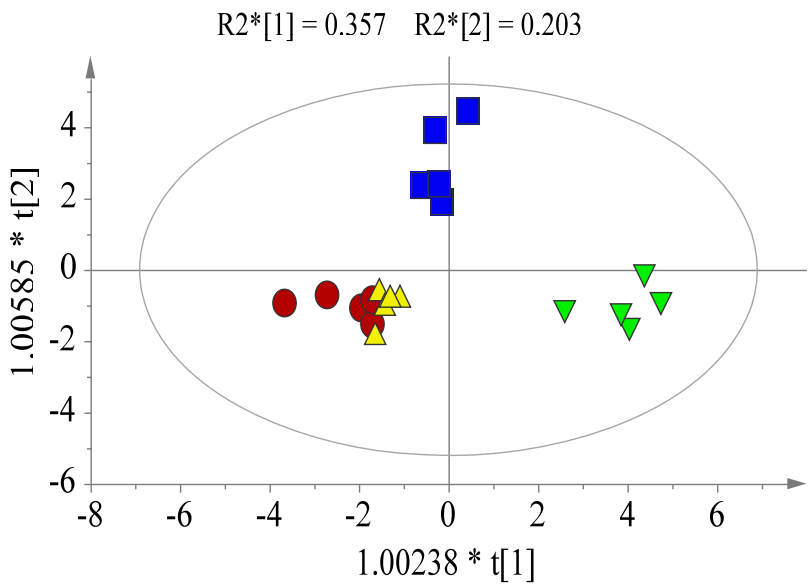

(b) OPLS-DA

Fig. 1 PCA and OPLS-DA analysis scores plot for different treatment of reed samples

From Figure 1(a), the results of PCA show that the reed samples were discrete obviously under different treatment conditions, however, the samples treated by 6days, not discrete very well, for further differentiating the samples of 6days treatment, the data was analyzed by OPLS-DA, the results were shown in Figure 1(b), the samples are more slightly discrete than that proceeded by PCA. The samples of 3days treatment exhibit more gather of separation. The $\mathrm{R} 2 \mathrm{Y}(\mathrm{cum})=0.849$ and $\mathrm{Q} 2(\mathrm{cum})=0.743$ are all more than 0.5 , which means the model is feasible.

\subsection{Metabolite Analysis}

OPLS-DA Loading diagram, VIP prediction diagram and analysis of variance were employed to find the potential biological markers at different treatment conditions. The OPLS-DA Loading diagram and VIP prediction diagram are shown in Figure 2 and Figure3. The potential biological markers are shown in Table 1. 


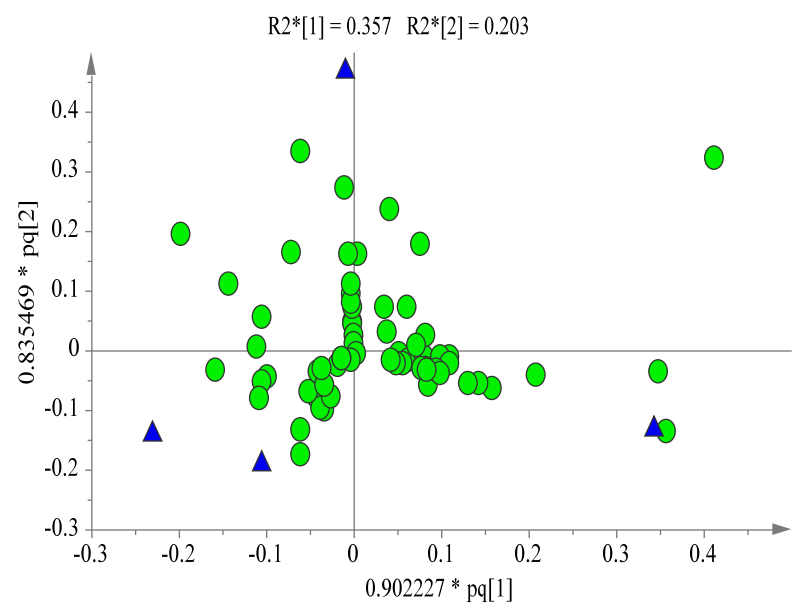

(a) OPLS-DA loading plots

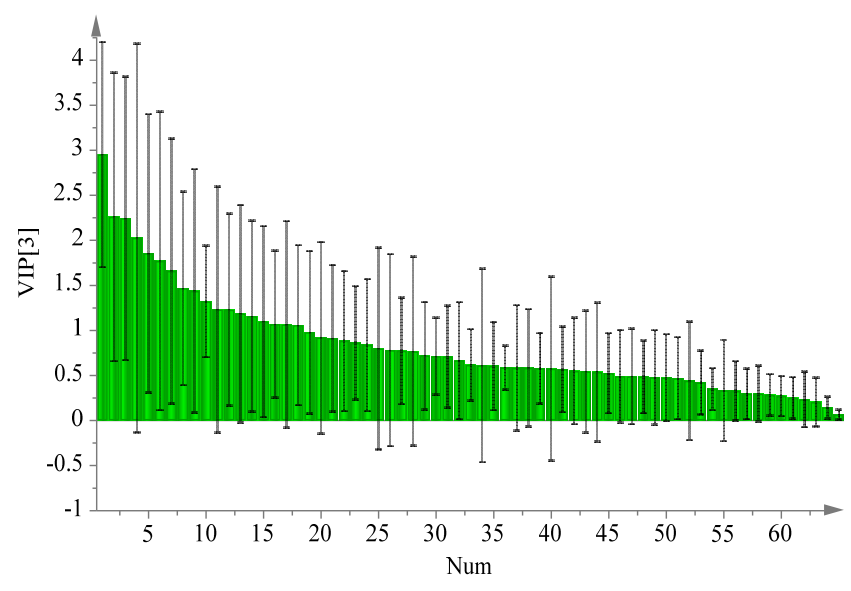

(b) VIP prediction plots

root exudates viable, $\boldsymbol{\Delta}$ samples group viable

Fig. 2 OPLS-DA analysis loading plots and VIP plots for root exudates of reed samples

Table 2. Statistical analysis results of the main metabolite changes in reed $(n=5)$

\begin{tabular}{|c|c|c|c|c|c|}
\hline & & Day 3 & & Day 6 & \\
\hline ID & Identity & -BPA & $+\mathrm{BPA}$ & -BPA & $+\mathrm{BPA}$ \\
\hline 31 & D-Glucopyranose & $1.00 \pm 0.01$ & $2.45 \pm 0.27$ & $2.42 \pm 0.46$ & $3.70 \pm 0.30$ \\
\hline 42 & Sitosterol & $1.00 \pm 0.83$ & $0.27 \pm 0.01$ & $0.58 \pm 0.56$ & $0.62 \pm 0.40$ \\
\hline 40 & D-Turanose & $1.00 \pm 0.52$ & $0.00 \pm 0.00$ & $0.00 \pm 0.00$ & $0.00 \pm 0.00$ \\
\hline 28 & D-Mannose & $1.00 \pm 0.55$ & $0.38 \pm 0.02$ & $0.08 \pm 0.00$ & $0.00 \pm 0.00$ \\
\hline 5 & Butanoic acid & $1.00 \pm 0.46$ & $0.12 \pm 0.00$ & $0.07 \pm 0.02$ & $0.16 \pm 0.16$ \\
\hline 37 & Octadecanoic acid & $1.00 \pm 0.02$ & $1.83 \pm 0.44$ & $1.55 \pm 0.52$ & $1.81 \pm 0.29$ \\
\hline 27 & Phthalic acid & $1.00 \pm 0.53$ & $0.24 \pm 0.04$ & $0.51 \pm 0.34$ & $0.52 \pm 0.39$ \\
\hline 26 & D-Fructose & $1.00 \pm 0.40$ & $4.98 \pm 0.48$ & $1.23 \pm 0.45$ & $1.46 \pm 0.33$ \\
\hline 15 & Nonanoic acid & $1.00 \pm 0.02$ & $0.00 \pm 0.00$ & $9.67 \pm 0.27$ & $0.00 \pm 0.00$ \\
\hline 19 & Aspartic acid & $1.00 \pm 0.02$ & $0.00 \pm 0.00$ & $0.00 \pm 0.00$ & $0.00 \pm 0.00$ \\
\hline 35 & Heptadecanoic acid & $0.00 \pm 0.00$ & $0.14 \pm 0.14$ & $0.00 \pm 0.00$ & $0.00 \pm 0.00$ \\
\hline 8 & Pentadecanoic acid & $1.00 \pm 0.01$ & $0.00 \pm 0.00$ & $8.77 \pm 0.37$ & $0.00 \pm 0.00$ \\
\hline 60 & Tetradecanoic acid & $0.00 \pm 0.00$ & $0.84 \pm 0.30$ & $0.61 \pm 0.36$ & $0.62 \pm 0.24$ \\
\hline 1 & Propanoic acid & $1.00 \pm 0.44$ & $0.26 \pm 0.18$ & $3.08 \pm 0.39$ & $4.17 \pm 0.00$ \\
\hline
\end{tabular}

Marks: The ratio of peak area of compounds with the same retention time at the different treatment conditions was standardized and expressed by average value \pm standard deviation.

The root exudates is very important for stabilizing the soil environment, and contacting soil bacteria[11][1]. The variation of reed exudates with the BPA stress will be used to explain the mechanism of reed tolerance and degradation of BPA. The 14 metabolites were found in the reed exudates, including: glucose, organic acids, amino acids and alcohols. The concentration of DGlucopyranose, Octadecanoic acid and Propanoic acid increased with the BPA treatment time and BPA concentration increase; The increase of D-Glucopyranose is benefit to enhance the activity of root bacteria concerning the BPA degradation[15][16][17].

The content of D-Glucopyranose, Heptadecanoic acid and Nutmeg acid are far more higher than that of control group, however, after 6days treatment with BPA, the content of three compounds above are far more lower than that treated by BPA for 3days. The phenomenon was also observed by Suzuk[i11], and explained that BPA degradation is accelerated by D-Glucopyranose, Heptadecanoic acid and Nutmeg acid released by reed root at very beginning, however, after 3days, with the treatment going on, these three compounds were reduced to increase the tolerance of the reed root.

The content of D-mucopolysaccharide, d-mannose, glusterol, butyric acid, benzoic acid and aspartic acid decreased with the treatment time and BPA concentration, the addition of BPA inhibited 
the secretion of D-mucopolysaccharide and aspartic acid, when the BPA treatment time reached 6days, the D-mucopolysaccharide secretion stopping, which means the reed is response to the BPA stress and reduced the release of the 6 compounds mentioned above.

Without the BPA addition, Nonanoic acid and 15-octane acid are very sensitive to the treatment time, with treatment time increase, the content of Nonanoic acid and 15-octane acid also increase; With the BPA addition, the Nonanoic acid and 15-octane acid were not detected in the samples, which means the BPA inhibited the secretion of these two compound, and then affect the normal growth of reed.

\section{Conclusion}

Plants of reed wetland was used as the research subject, basing on the Gas chromatography-mass spectrometry (GC-MS) analysis, metabonomics method was employed to detect and analyze the exudates of reed root. PCA and OPLS-DA were used to identify the main potential metabolites, these metabolites will be used to explore the mechanism of BPA tolerance and degradation of reed.

The conclusion is given as follows:

With and without BPA addition, the exudates of reed root was detected, 64 compounds were found, which include a variety of small molecule glucose (such as: RNA, fructose and mannose, etc.), organic acid (such as malic acid, succinic acid, etc.), amino acid (such as: L - valine, L- tyrosine, Lalanine, etc.), alcohols (such as: sorbitol, xylitol, inositol, etc.), and other small molecule compounds, such as Oleic acid, palmitic acid, dehydroabietic acid, etc.

The detection data was input into the SIMCA-P software, the metabolite secretion of reed was analyzed by PCA, OPLS-DA, VIP and ANOVA, the main metabolites with relationship to the tolerance and degradation of BPA. 14 compounds were found, mainly including glucose, organic acids, amino acids and alcohols, such as: d-glucose, D- mannose, sitosterol, D- pine disaccharide, butyric acid, stearic acid, the development of acid, D-fructose, pelargonic acid, aspartic acid, heptadecanoic acid, pentadecane acid, myristic acid, propionic acid.

\section{References}

[1]. Baetz U, Martinoia E. Root exudates: the hidden part of plant defense. Trends in plant science, 2014, 19(2): 90-98.

[2]. Li X, Ding C, Hua K, et al. Soil sickness of peanuts is attributable to modifications in soil microbes induced by peanut root exudates rather than to direct allelopathy. Soil Biology and Biochemistry, 2014, 78: 149-159.

[3]. Xie X, Liao M, Yang J, et al. Influence of root-exudates concentration on pyrene degradation and soil microbial characteristics in pyrene contaminated soil. Chemosphere, 2012, 88(10): 11901195.

[4]. Zhou N, Liu P, Wang Z Y, et al. The effects of rapeseed root exudates on the forms of aluminum in aluminum stressed rhizosphere soil. Crop Protection, 2011, 30(6): 631-636.

[5]. Phillips L A, Greer C W, Farrell R E, et al. Plant root exudates impact the hydrocarbon degradation potential of a weathered-hydrocarbon contaminated soil. Applied soil ecology, 2012, 52: 56-64.

[6]. Yang J, Pan X. Root exudates from sunflower (Helianthus annuus L.) show a strong adsorption ability toward Cd (II). Journal of Plant Interactions, 2013, 8(3): 263-270.

[7]. Jia X, Wang W, Chen Z, et al. Concentrations of secondary metabolites in tissues and root exudates of wheat seedlings changed under elevated atmospheric $\mathrm{CO} 2$ and cadmiumcontaminated soils. Environmental and Experimental Botany, 2014, 107: 134-143. 
[8]. Huang xin, Research on liver toxicology and data processing basing on GC-MS of metabonomics, [M]. Zhengjiang:medical school of Zhejiang University, 2008.

[9]. Qing LUO, Li-Na SUN, Xiao-Min HU. Metabonomics Study on Root Exudates of Cadmium Hyper.

[10]. Saito K., Hirai M.Y., Yonekura-Sakakibara K. Decoding genes with co-expression networks and metabolomics-'majority report by precogs'. Trends in plant science, 2008, 13(1): 36-43.

[11]. Suzuki K, Okazaki K, Tawaraya K, et al. Gas chromatography-mass spectrometry associated global analysis of rice root exudates under aseptical conditions. Soil Science and Plant Nutrition, 2009, 55(4): 505-513.

[12]. Duan Lixin, Qi Xiaoquan. Research on plant metabolomics basing on GC-MS. Life Science, 2015, 27(8): 971-977.

[13]. Lisec J, Schauer N, Kopka J, et al. Gas chromatography mass spectrometry-based metabolite profiling in plants. Nature protocols, 2006, 1(1): 387-396.

[14]. Gu Q., David F., Lynen F., etal. Evaluation of automated sample preparation, retention time locked gas chromatography-mass spectrometry and data analysis methods for the metabolomic study of Arabidopsis species. Journal of Chromatography A, 2011, 1218(21): 3247-3254.

[15]. Liu Y., Li X., Cai K., et al. Identification of benzoic acid and 3-phenylpropanoic acid in tobacco root exudates and their role in the growth of rhizosphere microorganisms. Applied Soil Ecology, 2015, 93: 78-87.

[16]. Zahar Haichar F, Santaella C, Heulin T, et al. Root exudates mediated interactions belowground. Soil Biology and Biochemistry, 2014, 77: 69-80.

[17]. Shi S, Richardson A E, O'Callaghan M, et al. Effects of selected root exudate components on soil bacterial communities. FEMS microbiology ecology, 2011, 77(3): 600-610. 